\title{
REVIEW
}

\section{Microsurgical vasovasostomy}

\author{
Lindsey Herrel and Wayland Hsiao
}

Up to $6 \%$ of men who have undergone vasectomy will ultimately elect for reversal in the form of vasovasostomy or vasoepididymostomy for various reasons. Vasovasostomy performed to regain fertility is a technique that has undergone numerous advances during the last century, including the use of microsurgical equipment and principles to construct a meticulous anastomosis. It is important during vasovasostomy to ensure good blood supply to the anastomosis as well as to build as a tension-free anastomosis. Visual inspection to ensure healthy mucosa and inner muscularis as well as atraumatic handling of tissues is helpful. With vasovasostomy, it is essential to creat a watertight anastomosis to prevent secondary scar formation. The microdot technique of vasovasostomy allows for markedly discrepant lumens to be brought together more precisely. Thereby, the planning is separated from suture placement, which prevents dog-ears and avoids subsequent leaks. In the age of in vitro fertilization (IVF)/intracytoplasmic sperm injection (ICSI), it becomes even more important to clarify outcomes after vasectomy reversals, as patients now have a choice between surgical sperm retrieval coupled with IVF/ICSI versus vasectomy reversal. Little data on long-term outcomes for vasectomy reversals exist. Therefore, further research in this field needs to evaluate the rate of late failures and the predictors of late failures.

Asian Journal of Andrology (2013) 15, 44-48; doi:10.1038/aja.2012.79; published online 12 November 2012

Keywords: male infertility; microsurgery; vasectomy; vasectomy reversal; vasovasostomy

\section{HISTORY}

Vasectomy as a form of surgical contraception has a long and vibrant history. Sir Astley Cooper was the first to perform vasectomy on a dog in 1823 , while Harrison performed the first vasectomy in a man for the treatment of benign prostatic hypertrophy. Vasectomy, as a contraceptive technique, has gained popularity, and today 175 000-354 000 vasectomies are performed in the United States each year. ${ }^{1}$ However, it is estimated that up to $6 \%$ of patients who undergo vasectomy ultimately elect for a reversal procedure. ${ }^{2}$

Edward Martin was Chief Surgeon at the University of Pennsylvania and is viewed as the Father of Modern Clinical Andrology. ${ }^{3,4} \mathrm{He}$ performed the first vasoepididymostomy on a patient with obstruction secondary to epididymitis in 1902 with reported patency rates of approximately $60 \%$. Additionally, he performed vasovasostomies on dogs. ${ }^{5}$ In 1919, William C. Quinby ${ }^{6}$ reported the first successful vasovasostomy in a human. During the mid twentieth century macroscopic vasovasostomies gained popularity. However, Sherman Silber $^{7}$ and Earl Owen ${ }^{8}$ ushered in the modern era of microsurgical vasectomy reversal with their independent introduction of microsurgical vasovasostomy in 1977.

Indications for a vasovasostomy include desire to have more children (remarriage or after death of a child), treatment of post vasectomy pain, or treatment of obstructive azoospermia due to traumatic or iatrogenic vasal injury (such as during hernia repair, orchidopexy or hydrocelectomy).

\section{ANATOMY}

The relevant gross anatomy to consider during vasovasostomy includes the vas deferens, epididymis and testis. Spermatogenesis begins in the seminiferous tubules. Sperm then travel to the rete testis and into the epididymis via the ductus efferentes. After transit through the caput, corpus and cauda of the epididymis, sperm are transported to the convoluted portion of the vas deferens followed by the straight portion of the vas deferens. It is in the epididymis that sperm mature and obtain motility. Finally, sperm are transported to the ejaculatory ducts where they are admixed with prostatic and seminal fluids.

The blood supply to the vas deferens derives from the deferential artery, a branch of the internal iliac artery from the aorta, as well as the inferior epididymal artery. During any operation involving the testis or vas, one must remember the blood supply in this area. Epididymal blood flow comes from the testicular artery (superior epididymal artery) and the deferential artery (inferior epididymal artery). Blood flow to the testis has origin in the testicular (aorta), deferential (internal iliac) and cremasteric arteries (inferior hypogastric). Inadvertent damage to the blood supply, particularly in patients with prior inguinal surgery, can cause testicular atrophy.

Histologically, the vas deferens is defined by three distinct layers. The innermost mucosal layer surrounds the lumen and is composed of pseudostratified columnar cells that are ciliated resting on a layer of basal cells. The second histological layer is the muscularis, which is composed of an inner and outer longitudinal layer with a middle circular layer. Finally, the vasal sheath surrounds and invests the vas itself and it is in this layer that the vasal arteries and vasal veins are found (Figure 1).

\section{PREOPERATIVE EVALUATION}

Prior to proceeding with surgical intervention, a complete history and physical exam should be performed. A comprehensive past medical history will help ensure that the patient is an appropriate operative candidate. A complete family history and any signs or symptoms consistent with hypogonadism should also be included. Additionally, important 


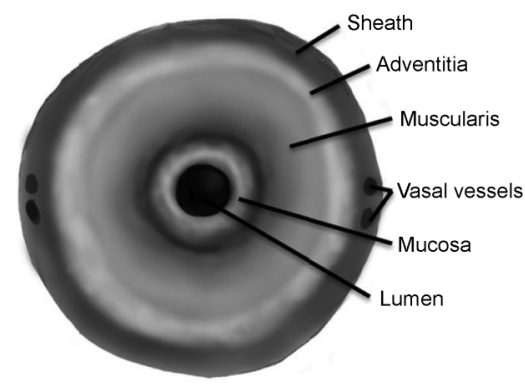

Figure 1 Diagram showing the three histological layers of the vas as well as the investing sheath.

information to gather includes the time since vasectomy, any prior inguinal or scrotal surgery, the age of the partner and any potential female factors contributing to infertility. A thorough reproductive history including any prior pregnancies with the current or a previous partner that have been achieved should be noted, since it has been demonstrated that vasectomy reversal with the same partner with a history of previous fertility leads to better outcomes. ${ }^{9}$ For patients with infertility prior to vasectomy, a testicular biopsy may be done either as a separate procedure prior to reversal or as the first step of the reversal procedure.

Physical exam will evaluate the general health of the patient, but should focus on the genital exam in particular. Examination of prior surgical scars and palpating the vasal gap and length of the testicular end remnant will aid in surgical planning. Previous scrotal surgeries, large vasal gaps or short testicular end remnants may raise the surgeon's suspicion that a more complex surgery may be needed. In addition, volume and consistency of the testes are important to note as patients with impaired spermatogenesis frequently have small, soft testes. An indurated or irregular epididymis may represent secondary epididymal obstruction and is more consistent with obstructive azoospermia than non-obstructive azoospermia. The presence sperm granuloma should be assessed. A sperm granuloma is thought to represent a 'pop off valve' after vasectomy due to sperm extravasation and may suggest a lower pressure in the testis and epididymal tubules. The proven predictive value of the presence or absence of a sperm granuloma is not yet known; however, some feel that there is a better prognosis of restored fertility after vasovasostomy when a sperm granuloma is present. ${ }^{10,11}$

Laboratory investigation can be useful in evaluating vasovasostomy candidates. A single semen analysis can rule out the remote possibility of recanalization of the vas. In fact, Lemack and Goldstein ${ }^{12}$ confirmed rare intact sperm on centrifuged semen pellets in $10 \%$ of men desiring a vasectomy reversal. The presence of sperm predicts a favorable prognosis for restored fertility after vasectomy reversal. Cryptospermia (defined as sperm seen on centrifuged pellet but not in initial semen analysis) in this setting was found to confer a favorable prognosis after vasectomy reversal. In patients with a low semen volume, a transrectal ultrasound can be considered to rule out ejaculatory duct obstruction. However, no routine preoperative imaging is indicated in the average vasectomy reversal patient. Recently, Lee et al. ${ }^{13}$ from Cornell showed that the presence of serum anti-sperm antibodies in a man is highly suggestive of active spermatogenesis. However, the clinical significance of these anti-sperm antibodies is unknown and it remains to be seen whether this is a useful test to obtain preoperatively. Finally, we believe that serum follicle-stimulating hormone should be drawn to evaluate for primary testicular failure, as elevated follicle-stimulating hormone level prior to vasectomy reversal may suggest a higher rate of utilization of assisted reproduction after vasectomy reversal. ${ }^{14}$

\section{ANESTHETIC}

Vasovasostomy is most commonly performed under general anesthesia. This provides the most favorable environment for the surgeon to create the optimal microsurgical anastomosis especially if more complicated repairs are necessary. Regional anesthetic, such as spinal or epidural anesthesia, can be used if long acting agents are available. We believe that local anesthesia only is a suboptimal anesthetic choice, as the operating microscope will magnify small patient movements, making successful repair more challenging. Only in patients who have a short interval to reversal, easily palpable vasal ends and are cooperative and free of anxiety should local anesthetic be considered. Once anesthetized, care must be taken to properly position and pad the patient to avoid neuromuscular injuries, which may result during a prolonged operation.

\section{INCISION OPTIONS}

The patient should be placed in the supine position with proper padding placed. The external ring should be marked prior to making the incision in all cases. The most frequently performed incision is high bilateral vertical scrotal incisions. Generally, this incision is made approximately $1 \mathrm{~cm}$ lateral to the base of the penis. This approach provides appropriate access in most cases of vasectomy reversals. Extension of this incision toward the external ring can be easily undertaken when necessary. Delivery of the testis, which this incision easily provides, allows for access to the entirety of the scrotal portion of the vas as well as the epididymis if necessary. Alternatively, an infrapubic incision can be made if the abdominal end of the vas is particularly short. There is little advantage to this incision over a high scrotal incision with inguinal extension. If suspicion is raised that the level of obstruction is within the inguinal vas, such as in patients with prior orchiopexy or herniorrhaphy, an inguinal incision through the previous incision can be used. Again, delivery of the testis into the field will allow for surgical access to the scrotal portion of the vas as well as the epididymis. Despite the type of incision, the surgeon must bear in mind the amount of tension that will be placed on the anastomosis when exposed portions are returned to their natural anatomic position. A final option to consider is the no-scalpel technique pioneered by Jarvi et al., ${ }^{15}$ which minimizes the length of incision. During this approach, the vas is grasped with a cantilevered C-clamp through the skin and the no-scalpel vasectomy hemostat is used to puncture the skin and underlying dartos fascia. The $\mathrm{C}$-clamp is then used to bring the vas deferens to the skin surface. Once both ends of the vas have been brought through the incision, the standard anastomosis is performed.

\section{PROPERTIES OF A SUCCESSFUL ANASTOMOSIS}

There are a number of principles, which will ensure a healthy anastomosis and successful outcomes. It is important to adhere to several basic tenets when constructing an anastomosis for vasectomy reversal. The first of these principles is ensuring a good blood supply to the tissues to be anastomosed. Handling the tissue in an atraumatic fashion is of critical importance to maintain the health and blood supply of the tissue. Additionally, the areas to be anastomosed should be visually inspected to ensure healthy mucosa and muscularis. For the anastomosis itself, accurately approximation of mucosa to mucosa is paramount. Next, the creation of a watertight anastomosis will prevent secondary scar formation. Finally, a tension-free anastomosis should be constructed.

\section{INSTRUMENTS USED}

As with any specialized surgery, dedicated equipment is of utmost importance. First, the use of the operating microscope is mandatory. Sitting during the operation is preferred to reduce surgeon fatigue and improve fine motion. This is aided by the use of armrests. We find that the use of the Goldstein Microspike Approximator clamp (ASSI Corp., 
NY, USA). ${ }^{16}$ greatly facilitates the anastomosis by holding the two vasal ends in proximity and stabilizing the vasal ends. Additionally, this clamp helps prevent tension on the anastomosis. A slotted nerve clamp and a flat microsurgical ultrasharp knife allow precise $90^{\circ}$ amputation of the vas for anastomosis. If a no-scalpel technique is utilized, a sharpened hemostat and cantilevered C-clamp is useful to bring the vas to the skin surface. Bipolar cautery is preferred to monopolar cautery to prevent thermal injury to surrounding tissues.

\section{PREPARING THE VASA}

Once the incision is made, the first step is to prepare the vas and gain adequate vasal length for a tension-free anastomosis. Once the previous vasectomy site is identified, attempts are made to mobilize the vas both towards the testis as well as towards the external inguinal ring. Mobilization can be performed bluntly with a gauze-covered fingertip or kittner. It is important to avoid stripping the outer vasal sheath, which will prevent disruption of the blood supply. When mobilizing vasal ends, it is important to avoid ligating any vessels that may supply the vas. Transillumation of perivasal tissues may be exceedingly useful in this setting. This enables better identification and preservation of vessels going to the vas itself.

Adequate vasal length should be one of the earliest goals of vas mobilization. If there is insufficient vasal length, the incision can be extended toward the external ring to allow more dissection at the abdominal end. Additional options for obtaining vasal length include dissecting the convoluted vas off of the testis for length and dissection of the vasoepididymal junction off the testis thereby freeing the epididymal tail.

The vasal ends should then be cut at a $90^{\circ}$ angle to allow for precise anastomosis. This can be done utilizing a slotted nerve clamp to hold the vas and cutting the end with an ultrasharp knife. Once cut, each end is inspected for a healthy mucosa (which springs back when gently touched with a jeweler's forcep), smooth muscularis and healthy bleeding tissue. The vasal artery and vein can be ligated with 6-0 nylon if bleeding is going to interfere with construction of the anastomosis.

Next, the patency of the abdominal vasal end should be assessed. This can be accomplished in a number of different ways. The first method is to intubate the abdominal end and secure a watertight seal with a 24-gauge angiocatheter on a $1 \mathrm{ml}$ syringe filled with saline. Gently, the saline is injected; if there is no resistance and the fluid does not return, then patency can be assured. If this fails, a 16-French Foley catheter is placed with $5 \mathrm{ml}$ filled into the balloon and pulled gently against the bladder neck. Then $1 \mathrm{ml}$ of indigo carmine is injected into the abdominal end of the vas using a 24-gauge angiocatheter. If the urine turns blue, patency is confirmed. A third option is to pass a 2-0 prolene suture using the non-needle end into the abdominal end. If this passes easily, patency is established. If the suture does not pass easily, the distance to obstruction can be measured based on the length of suture passed prior to meeting resistance. Finally, a formal vasogram can be obtained utilizing water-soluble contrast and a flat plate X-ray.

Next, the testicular end of the vas should be evaluated for the presence of sperm. The vasal fluid from the testicular end should be collected on a glass slide for immediate microscopic evaluation. If no fluid is collectable, a 24-gauge angiocatheter filled with saline can be inserted into the testicular vasal end and a gentle barbotage can be obtained and the fluid examined for sperm. Table 1 shows the possible fluid findings and corresponding suggested procedure. The technique of vasoepididymostomy will be covered in another chapter in current Special Issue.

\section{ONE-LAYER TECHNIQUE}

Benefits of a one-layer technique are its simplicity, relative speed with which it can be performed, and the requirement for less microsurgical training. The disadvantage to this technique is that it does not account for discrepant lumen sizes, which theoretically may lead to lower patency rates. In the true one-layer anastomosis, a full-thickness suture is placed through all layers of the vas to bring the two ends together. In the modified one layer, an additional layer of interrupted seromuscular sutures is placed in between the full thickness sutures. ${ }^{17}$ Despite the relative simplicity of these techniques, formal laboratory microsurgical training remains imperative.

\section{TWO-LAYER TECHNIQUE}

During a two-layer technique, an inner layer of sutures is placed to bring the mucosa together. Next, an outer layer of sutures closing the seromuscular layers is placed. This has been compared to the traditional one-layer technique, and demonstrated similar outcomes as shown by the VV study group. ${ }^{11}$ To perform a two-layer anastomosis, more dedicated microscopic training is required. Theoretically, an additional layer should help approximate discrepant lumens and allow for a more watertight anastomosis.

\section{MICRODOT MULTILAYER TECHNIQUE}

Goldstein et al. ${ }^{18}$ first described the microdot technique in 1998 at Weill Cornell Medical College. This technique allows for markedly discrepant lumens to be brought together more precisely. Utilizing the microdot technique, the planning is separated from suture placement. This presuture placement mapping prevents dog-ears and subsequent leaks can be avoided. Once the vasa are prepared, a microtip (Covidien, Mansfield, MA, USA), marking pen is utilized to define the needle exit points. A total of six 'microdots' are placed on the cut ends of the vas, approximately one-third of the distance between the lumen and outer adventitia on the muscularis. The first mucosal layer is placed utilizing the microdots and 10-0 monofilament suture. Use of a double-armed suture can prevent back walling of the vas lumen. The second layer approximates the deep muscularis layer with a 9-0 monofilament suture. Care is taken with this second layer not to incorporate the mucosal layer or inadvertently close off the lumen. The third layer closes the adventitial layer in a watertight fashion with a 9-0 monofilament suture. All sutures placed are in an interrupted fashion. Finally, the vasal sheath is re-approximated using six 9-0 nylons or 7-0 prolene which reduces tension on the anastomosis (Figure 2).

\section{SPECIAL CONSIDERATIONS}

Occasionally, large vasal gaps can be encountered during vasovasostomy. There are several options for gaining vasal remnant length. The first technique is to bluntly dissect to free the abdominal end (which can be done well within the inguinal canal) as well as freeing the testicular end of the vas. This can be done with gauze over the tip of the surgeon's finger or a kittner, taking care not to strip off the vasal sheath. Next, the vasoepididymal junction can be dissected to reflect the epididymis off the testis. This can be done to free approximately

\section{Table 1 Vasal fluid findings}

\begin{tabular}{|c|c|c|}
\hline $\begin{array}{l}\text { Gross vasal fluid } \\
\text { characteristics }\end{array}$ & Microscopic findings & $\begin{array}{l}\text { Recommended } \\
\text { procedure }\end{array}$ \\
\hline \multirow[t]{4}{*}{ Clear, cloudy or creamy fluid } & Motile sperm & Vasovasostomy \\
\hline & Whole sperm, non-motile & Vasovasostomy \\
\hline & $\begin{array}{l}\text { Sperm heads and whole } \\
\text { non-motile sperm }\end{array}$ & Vasovasostomy \\
\hline & Sperm heads only & Vasovasostomy \\
\hline Copious clear fluid & No sperm on barbotage & Vasovasostomy \\
\hline $\begin{array}{l}\text { Creamy or tooth } \\
\text { paste-like fluid }\end{array}$ & No sperm on barbotage & Vasoepididymostomy \\
\hline No fluid, no granuloma & No sperm on barbotage & Vasoepididymostomy \\
\hline
\end{tabular}


a

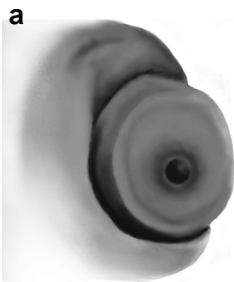

d

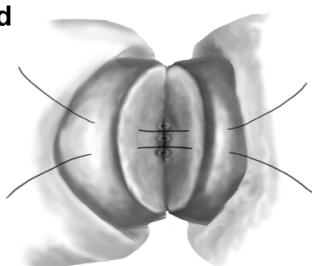

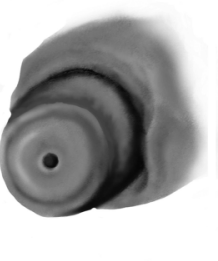

e

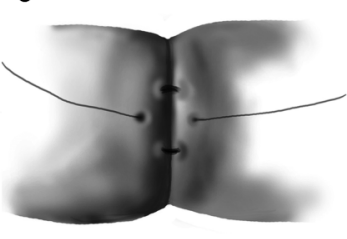

b

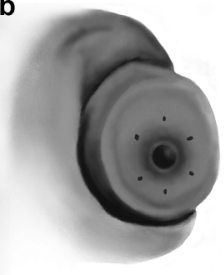

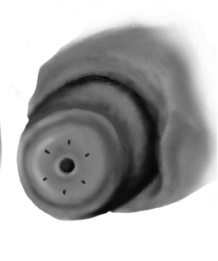

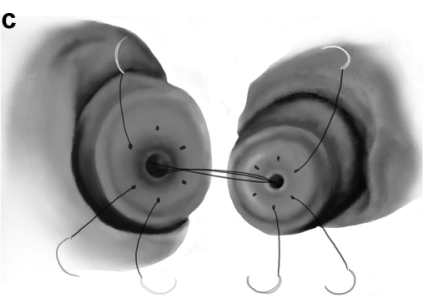

g

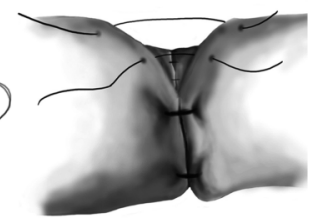

Figure 2 Microdot vasovasostomy. (a) Discrepant lumens sizes between the abdominal and testicular ends are shown. (b) Microdots are made approximately onethird of the way between the lumen and the adventitial layer. (c) Double-arm 10-0 nylon sutures are passed precisely through three microdots. (d) 9-0 nylon sutures are placed exactly between the 10-0 mucosal sutures. (e) A third layer of 9-0 nylon sutures are placed between the 9-0 sutures in the second layer and directly overlying the 10-0 mucosal layer. (f) The anastomosis is flipped and an identical procedure is performed on the other side starting with three double-armed 10-0 nylon sutures. (g) Diagram showing the final fourth layer reapproximating the vasal sheath for less tension on the anastomosis.

one-half to two-thirds of the length of the epididymis. A third option is to perform an orchidopexy to position the testis higher within the scrotum. The testis can also be positioned in a horizontal position or even upside down if necessary. In cases where the testicular end is shortened, anastomosis can be performed to the convoluted vas with similar outcomes. ${ }^{19}$ If anastomosis must be made to the convoluted vas, care must be taken to ensure that both ends are cut at a $90^{\circ}$ angle. If the convoluted end is cut at an obtuse angle, the anastomosis will not be lined up properly and will not be watertight (Figure 3). A final option to provide additional length on the abdominal end vas segment is to perform a laparoscopic mobilization of the intra-abdominal vas and re-route this segment medial to the epigastric vessels. A standard microdot vasovasostomy can then be performed in the groin.

When multiple vasal obstructions are encountered such as with a previous herniorrhaphy with vasectomy, considerations must be made because two vasovasostomies cannot be performed in tandem without compromising the intervening segment. Hence, when this circumstance is encountered, the intervening segment between the two obstruction sites must be removed. The previously mentioned techniques are then used to close the large vasal gap.

When a varicocele is encountered in patients preparing for vasectomy reversal, careful planning is necessary. Performing both procedures simultaneously could result in poor venous return and testis atrophy or loss. A thorough microsurgical varicocelectomy ligates all venous return from the testis with the exception of the vasal veins. When a vasectomy has been performed, preservation of some venous return must be maintained during varicocelectomy such as the cremasteric veins. Performing both procedures simultaneously could result in poor venous return and testis atrophy or loss. In a limited number of simultaneous vasovasostomy/varicocelectomy cases at Weill Cornell, cremasteric veins were intentionally not ligated to prevent testis atrophy. In this particular population, a varicocele recurrence rate of up to $22 \%$ was observed (Goldstein $\mathrm{M}$, personal communication). Ideally, the vasectomy reversal is completed approximately 6 months or more prior to varicocelectomy. Preservation of the testicular artery is critical when performing these procedures in tandem.

In patients who have inguinal vasal obstruction with contralateral absence of the testis, atrophy of the testis or epididymal obstruction, a crossed vasovasostomy can be performed. During this procedure, the compromised vas attached to the healthy testis is dissected as far as possible to gain length on the testicular remnant. This end is passed through the scrotal septum at the base of the penis through a wide tunnel. The abdominal end of the unobstructed vas attached to the unhealthy testis is dissected at the level of the junction of the straight and convoluted vas to obtain as much length as possible on both ends prior to anastomosis. A final method of closing a large vasal gap is to transpose the testis to the contralateral side of the scrotum and perform an orchidopexy. Once adequate length is obtained, a standard microdot vasovasostomy is performed.

\section{POSTOPERATIVE MANAGEMENT}

Patients are generally operated on in an outpatient setting and are discharged postoperatively. Instructions to wear a scrotal support for 6 weeks are given. Light activity is allowed starting 3 days postoperatively; however, no sports or heavy workload should be undertaken for 2 weeks and sexual activity should be avoided for 4 weeks total. Semen analyses

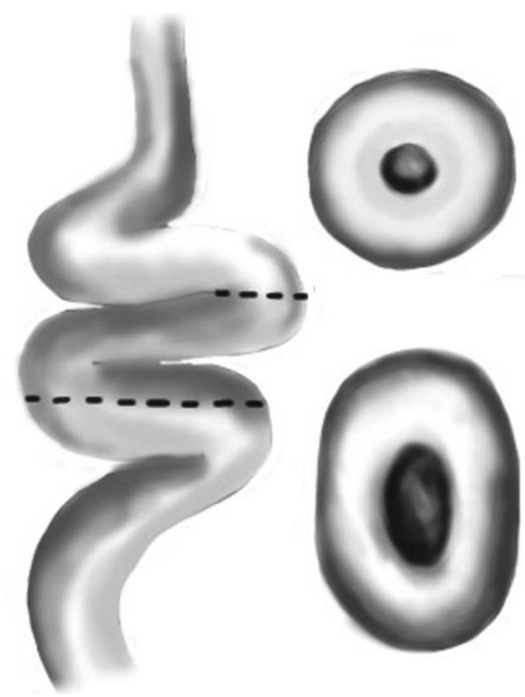

Figure 3 Figure showing the difference in the resulting lumen when you cut at a point in the convoluted vas at a point that is exactly perpendicular to the lumen (top figure) versus at an oblique point (bottom figure). 
are collected at 2, 4 and 6 months postoperatively. If azoospermia persists at 6 months, a revision of the vasovasostomy may be necessary.

\section{COMPLICATIONS}

Complications of vasovasostomy are rare. Formation of a scrotal hematoma remains the most common postoperative complication. A series of 2500 operations had seven occurrences of scrotal hematoma. ${ }^{20}$ None of these required surgical intervention and no wound infections were encountered. If a scrotal hematoma does form, it generally resolves in 6-12 weeks with conservative treatment. If extensive dissection is necessary, placement of Penrose drains in the dependent portion of the scrotum can help prevent hematoma formation. Drains are generally removed within $24 \mathrm{~h}$ after surgery.

Obstruction and recurrent stricture, or secondary azoospermia, are another potential long-term complications. Kolettis et al. ${ }^{21}$ reported a transient patency rate of $2.9 \%-5.3 \%$, depending on the intraoperative presence of sperm. The formation of a sperm granuloma postoperatively can indicate leakage of sperm at the site of anastomosis and is a predictor of failure. Once sperm are identified in the ejaculate, cryopreservation should be considered in the event future failure occurs.

\section{OUTCOMES}

In the age of in vitro fertilization (IVF)/intracytoplasmic sperm injection (ICSI), it becomes even more important to clarify outcomes after vasectomy reversals, as patients now have a choice between surgical sperm retrieval coupled with IVF/ICSI versus vasectomy reversal. The factors involved in this incredibly complex decision include (but are not limited to) the number of children desired, age of the female partner, male's past surgical history, a couples' financial resources, availability of an experienced microsurgeon, religious preferences of the couple and previous fertility together. One of the most comprehensive studies published on vasovasostomy outcomes is from Belker et al. of the Vasovasostomy Study Group. ${ }^{11}$ In this multicenter study, a patency rate of $86 \%$ and pregnancy rate of $52 \%$ were reported. Important factors contributing to patency were duration of obstructive interval and use of vasoepididymostomy. Obstructive interval, as well as the character of the vasal fluid present at the time of reversal, influenced pregnancy rates. One layer versus two layers and presence of sperm granuloma were not found to impact patency.

A brief review of the microsurgical vasectomy reversal literature reports patency rates ranging from $69.2 \%$ to $97.8 \%,{ }^{22,23}$ with pregnancy rates ranging from $36.8 \%$ to $92.5 \%{ }^{24,25}$ However, the rate of return of sperm to the ejaculate may vary depending on several factors. Yang et $a .^{26}$ found that motile sperm in the vasal fluid at the time of vasectomy reversal predicted faster return of sperm of the ejaculate after vasectomy reversal. In a study of 150 vasectomy reversals, $95 \%$ achieved patency within 6 months when motile sperm was seen intraoperatively, while only $76 \%$ of patients achieved patency within 6 months if motile sperm were not found. ${ }^{26}$ In addition, patency was achieved much sooner in those undergoing a vasovasostomy when compared to patients who only underwent vasoepididymostomy, with a rate of return of sperm to the ejaculate of up to 2 years with vasoepididymostomy. Reports of average sperm counts post-vasectomy reversal are poorly defined with varying definitions of success found in the literature.

However, previous papers on vasectomy reversal have generally examined a single variable when evaluating vasectomy reversal outcomes. Hsiao et al. ${ }^{27}$ have published a nomogram which takes into account age, duration of obstruction, prior reversal, presence of sperm granuloma, testis volume, procedure performed, fluid and presence of sperm seen intraoperatively and puts it into one model, with a nomogram generated for preoperative chance of patency and postoperative chance of patency. Using this model, we believe that patients can better make the decision whether to proceed with vasectomy reversal versus sperm retrieval and IVF/ICSI. In fact, the postoperative nomogram is helpful for counseling patients whether they should cryopreserve semen after vasectomy reversal.

Little data on long-term outcomes for vasectomy reversals exist. Therefore, further research in this field is needed to evaluate the rate of late failures and the predictors of late failures. Additionally, data on outcomes are very heterogeneous, which impairs consistent outcome reporting. We need more standardized reporting measures for these vasectomy reversal studies, to truly define measures of surgical success.

\section{ACKNOWLEDGMENTS}

We would like to acknowledge Vanessa Dudley for her assistance in the preparation of the figures used in this review.

\section{COMPETING FINANCIAL INTERESTS}

The authors would declare no competing financial interests.

1 Eisenberg ML, Lipshultz LI. Estimating the number of vasectomies performed annually in the United States: data from the National Survey of Family Growth. J Urol 2010; 184: 2068-72.

2 Sandlow JI, Nagler HM. Vasectomy and vasectomy reversal: important issues. Preface. Urol Clin North Am 2009; 36: xiii-xiv.

3 Martin E, Carnett JB, Levi JV, Pennington ME. The surgical treatment of sterility due to obstruction at the epididymis; together with a study of the morphology of human spermatozoa. Univ Pa Med Bull 1902; 15: 2-15.

$4 \mathrm{Kim} \mathrm{HH}$, Goldstein M. History of vasectomy reversal. Urol Clin North Am 2009; 36: 359-73.

5 Crain DS, Roberts JL, Amling CL. Practice patterns in vasectomy reversal surgery: results of a questionnaire study among practicing urologists. J Uro/ 2004; 171: 311-5.

6 O'Conor V. Anastomosis of vas deferens after purposeful division for sterility. J Am Med Assoc 1948; 136: 162.

7 Silber SJ. Microscopic vasectomy reversal. Fertil Steril 1977; 28: 1191-202.

8 Owen ER. Microsurgical vasovasostomy: a reliable vasectomy reversal. Aust NZ J Surg 1977; 47: 305-9.

9 Chan PT, Goldstein M. Superior outcomes of microsurgical vasectomy reversal in men with the same female partners. Fertil Steril 2004; 81: 1371-4.

10 Boorjian S, Lipkin M, Goldstein M. The impact of obstructive interval and sperm granuloma on outcome of vasectomy reversal. J Urol 2004; 171: 304-6.

11 Belker AM, Thomas AJ Jr, Fuchs EF, Konnak JW, Sharlip ID. Results of 1,469 microsurgical vasectomy reversals by the Vasovasostomy Study Group. J Urol 1991; 145: 505-11.

12 Lemack GE, Goldstein M. Presence of sperm in the pre-vasectomy reversal semen analysis: incidence and implications. J Urol 1996; 155: 167-9.

13 Lee R, Goldstein M, Ullery BW, Ehrlich J, Soares M et al. Value of serum antisperm antibodies in diagnosing obstructive azoospermia. J Urol 2009; 181: 264-9.

14 Hsiao W, Sultan R, Lee R, Goldstein M. Increased follicle-stimulating hormone is associated with higher assisted reproduction use after vasectomy reversal. J Urol 2011; 185: 2266-71.

15 Jarvi K, Grober ED, Lo KC, Patry G. Mini-incision microsurgical vasectomy reversal using no-scalpel vasectomy principles and instruments. Urology 2008; 72: 913-5.

16 Goldstein M. Microspike approximator for vasovasostomy. J Urol 1985; 134: 74

17 Sharlip I. Microsurgical vasovasostomy: modified one-layer technique. In: Goldstein M, editor. Surgery of Male Infertility. Philadelphia, PA: W.B. Saunders Company; 1995. p67-76.

18 Goldstein M, Li PS, Matthews GJ. Microsurgical vasovasostomy: the microdot technique of precision suture placement. J Urol 1998; 159: 188-90.

19 Sandlow JI, Kolettis PN. Vasovasostomy in the convoluted vas deferens: indications and outcomes. J Urol 2005; 173: 540-2.

20 Goldstein M. Surgical management of male infertility. In: Wein AJ, Kavoussi LR, Novick AC, Partin AW, Peters CA, editors. Campbell-Walsh Urology. 10th ed. Philadelphia, PA: Elsevier Saunders; 2012. p648-87.

21 Kolettis PN, Fretz P, Burns JR, D'Amico AM, Box LC et al. Secondary azoospermia after vasovasostomy. Urology 2005; 65: 968-71.

22 Ratana-Olarn K, Gojaseni P, Muangman V, Visethsin V, Ariyaprakai W et al. Vasectomy reversal: experience in Ramathibodi Hospital, Thailand. J Med Assoc Thai 1982; 65: 240-5.

23 Patel SR, Sigman M. Comparison of outcomes of vasovasostomy performed in the convoluted and straight vas deferens. J Urol 2008; 179: 256-9.

24 Chiang HS. Clinical study of vasectomy reversal: results of 60 single-surgeon cases in Taiwan. J Formos Med Assoc 1996; 95: 866-9.

25 Silber SJ, Grotjan HE. Microscopic vasectomy reversal 30 years later: a summary of 4010 cases by the same surgeon. J Androl 2004; 25: 845-59.

26 Yang G, Walsh TJ, Shefi S, Turek PJ. The kinetics of the return of motile sperm to the ejaculate after vasectomy reversal. J Urol 2007; 177: 2272-6.

27 Hsiao W, Goldstein M, Rosoff JS, Piccorelli A, Kattan MW et al. Nomograms to predict patency after microsurgical vasectomy reversal. J Urol 2012; 187: 607-12. 